\title{
REVISIÓN
}

\section{El refino de los metales del grupo del platino ${ }^{(\bullet)}$}

\begin{abstract}
F.J. Alguacil ${ }^{(*)}$
Resumen En un trabajo anterior se describieron los procesos que permiten obtener disoluciones libres de oro y abordar la recuperación de los metales del grupo del platino. En el presente trabajo se evalúan los procesos clásicos para el refino de estos metales. Asimismo, se discuten los nuevos procesos de refinación basados en la aplicación de la extracción con disolventes.
\end{abstract}

Palabras clave: Metales del grupo del platino. Extracción con disolventes. Recuperación. Refino.

\section{The refining of the platinum group metals}

\begin{abstract}
In a previous work the new processes from which free gold solutions can be obtained and thus allowing the recovery of platinum group metals has been described. Present work evaluates the classical processes for the refining of such metals. The new processes based on solvent extraction refining are also discussed.
\end{abstract}

Keywords: Platinum group of metals. Solvent extraction. Recovery. Refining.

\section{INTRODUCCIÓN}

Los nuevos procesos encaminados al refino del oro tienen como particularidad común que permiten la obtención de disoluciones libres de este metal y facilitan la recuperación posterior de los metales del grupo del platino (1). Tradicionalmente, estos metales se han recuperado a partir de sus propiedades químicas, especialmente, por la variación que presentan en sus estados de oxidación y por la coordinación de sus complejos en medio cloruro (2-4). La tabla I muestra estos clorocomplejos que, en la mayoría de los casos, son compuestos aniónicos, siendo difícil encontrar en este medio cloruro especies catiónicas. En algunos casos, sobre todo en los metales más pesados, se pueden encontrar complejos mixtos $\mathrm{Cl}^{-}-\mathrm{H}_{2} \mathrm{O}$ e, incluso, especies polinucleares.

Los complejos clorurados se diferencian en la mayor o menor facilidad con la que pueden presentar reacciones de sustitución; en términos generales, estas sustituciones son más lentas que en el caso de los metales base y siguen la secuencia: $\mathrm{Pd}(\mathrm{II})>$ $\mathrm{Pt}(\mathrm{II})>\mathrm{Ru}(\mathrm{III})>\mathrm{Rh}(\mathrm{III})>\operatorname{Ir}(\mathrm{III})>\mathrm{Os}(\mathrm{III})>\operatorname{Ir}(\mathrm{IV})$ y $\mathrm{Pt}(\mathrm{IV})$. Se considera que a partir del Rh(III) los

\footnotetext{
(•) Trabajo recibido el día 2 de noviembre de 1994.

(*) Centro Nacional de Investigaciones Metalúrgicas (CSIC). Avda. de Gregorio del Amo, 8. 28040-Madrid (España).
}

TABLA I.- Complejos clorurados más importantes de los metales del grupo del platino

TABLE I.-Common chlorocomplexes of the platinum group metals

\begin{tabular}{|c|c|c|c|}
\hline Metal & $\begin{array}{c}\text { Estado de } \\
\text { oxidación }\end{array}$ & Complejo & Estructura \\
\hline $\mathrm{Pt}$ & II & $\mathrm{PtCl}_{4}^{2-}$ \\
$\mathrm{IV}$ & $\begin{array}{c}\text { Plano cuadrada } \\
\text { Octaédrica }\end{array}$ \\
\hline $\mathrm{Pd}$ & II & $\begin{array}{c}\mathrm{PdCl}_{4}^{2-} \\
\mathrm{PdCl}_{6}^{2-}\end{array}$ & $\begin{array}{c}\text { Plano cuadrada } \\
\text { Octaédrica }\end{array}$ \\
\hline Ir & III & $\begin{array}{c}\mathrm{IrCl}_{6}^{3-} \\
\mathrm{IrCl}_{6}^{2-}\end{array}$ & $\begin{array}{c}\text { Octaédrica } \\
\text { Octaédrica }\end{array}$ \\
\hline $\mathrm{Rh}$ & III & $\mathrm{RhCl}_{6}^{3-}$ & Octaédrica \\
\hline $\mathrm{Ru}$ & III & $\begin{array}{c}\mathrm{RuCl}_{6}^{3-} \\
\mathrm{RuCl}_{6}^{2-}\end{array}$ & $\begin{array}{c}\text { Octaédrica } \\
\text { Octaédrica }\end{array}$ \\
\hline Os & III & $\begin{array}{c}\mathrm{OsCl}_{6}^{3-} \\
\mathrm{OsCl}_{6}^{2-}\end{array}$ & $\begin{array}{c}\text { Octaédrica } \\
\text { Octaédrica }\end{array}$ \\
\hline
\end{tabular}


estados de oxidación son inertes. Por lo tanto, es muy importante considerar los factores cinéticos, puesto que reacciones que termodinámicamente son posibles, luego, en la práctica, no presentan los mismos rendimientos o no son tan fáciles de llevar a cabo.

Estos procesos de refino, en general largos y complicados en su manipulación, están siendo reemplazados por otros nuevos, que en su mayoría están basados en la extracción con disolventes, aunque en aquellas refinerías en las que se tratan materiales con un elevado contenido de estos metales preciosos, los procesos clásicos todavía tienen su importancia.

\section{LOS PROCESOS CLÁSICOS PARA EL REFINO DE LOS METALES DEL GRUPO DEL PLATINO}

Generalmente, estos procesos se han basado en la recuperación, por un lado, del platino y el paladio, para a continuación abordar la recuperación de los metales secundarios de este grupo. Las figuras 1 y 2 muestran unos esquemas generales de tratamiento para estos dos grupos mencionados.

Como norma general, el concentrado que contiene a los metales preciosos se trata, con el fin de eliminar, tanto como sea posible, los metales base y la plata. La tendencia a ser inertes que presentan estos metales nobles hace que esta parte del proceso no sea realmente difícil, aunque ciertos metales tales como el estaño pueden complicar el esquema de tratamiento.

A continuación, la materia prima se lixivia con agua regia, de forma que el oro, platino y paladio pasan a la disolución. El oro, que es el más noble termodinámicamente de todos ellos, se recupera primero mediante una precipitación con un agente reductor y, después, se precipita el platino con cloruro amónico. Este último precipita como complejo de hexacloroplatinato de amonio, y el metal puro se obtiene a partir de este precipitado bien mediante una reducción, con ácido fórmico por ejemplo, y calcinación, o por una simple calcinación. Una sola operación de este tipo muy raramente da lugar a un material que sea comercializable, por lo que se necesitan varias operaciones de redisolución y reprecipitación hasta que se alcanza el grado de pureza necesario.

A partir de este punto se recupera el paladio de la disolución que no contiene platino. En primer lugar, se adiciona amoniaco hasta un $\mathrm{pH}$ aproximado de 8 , eliminándose los metales base, y a continuación, se acidifica con ácido clorhídrico hasta un valor de $\mathrm{pH} 1$, precipitando el cloruro de diaminpaladio(II). La adición de amoniaco se hace lenta-

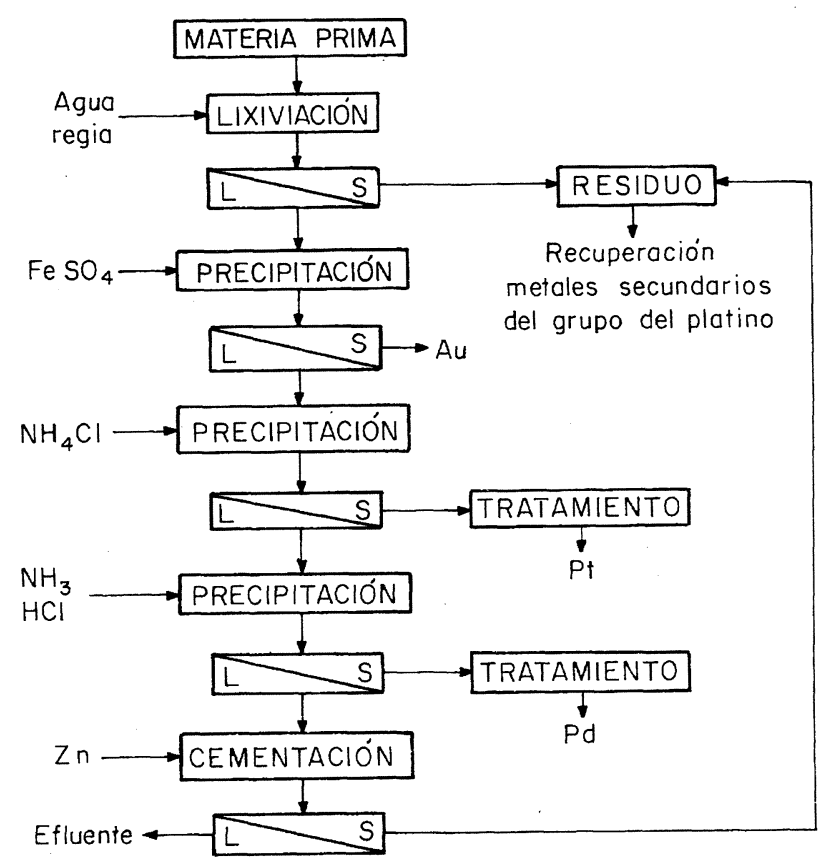

Fig. 1.- Esquema clásico para el refinado de los metales del grupo del platino.

FIG. 1.-Classical platinum group metals refining scheme.

mente, ya que la reacción es exotérmica y la temperatura alcanza los $60-80^{\circ} \mathrm{C}$.

El metal se recupera de la sal por una reducción y/o calcinación, aunque como en el caso del platino, se necesitan varias operaciones de redisolución y reprecipitación hasta alcanzar el grado de pureza necesario.

Existe alguna variación con respecto a este esquema de tratamiento $y$, así, se ha propuesto un proceso en el que el paladio se recupera como complejo de hexacloropaladato de amonio; es decir, el paladio se encuentra como $\mathrm{Pd}(\mathrm{IV})$. Este proceso no es tan selectivo como el mencionado anteriormente $\mathrm{y}$, además, la disolución obtenida después del proceso de precipitación todavía contiene entre 0,3 a $0,5 \mathrm{~g} / \mathrm{l}$ de estas sales, debido a su propia solubilidad, siendo necesaria la recuperación de estos valores residuales mediante cementación con cinc, reciclándose el cemento a la etapa de lixiviación con agua regia (5).

La recuperación de los metales secundarios del grupo del platino se lleva a cabo a partir del residuo de la lixiviación con agua regia del material de partida. Este residuo se funde con plomo metálico o con carbonato de plomo, de forma que tanto el oro, platino y paladio que eventualmente estuvieran en el residuo, se pueden lixiviar en un segundo tratamiento con agua regia, mientras que los elementos secundarios más refractarios quedan en el residuo. 


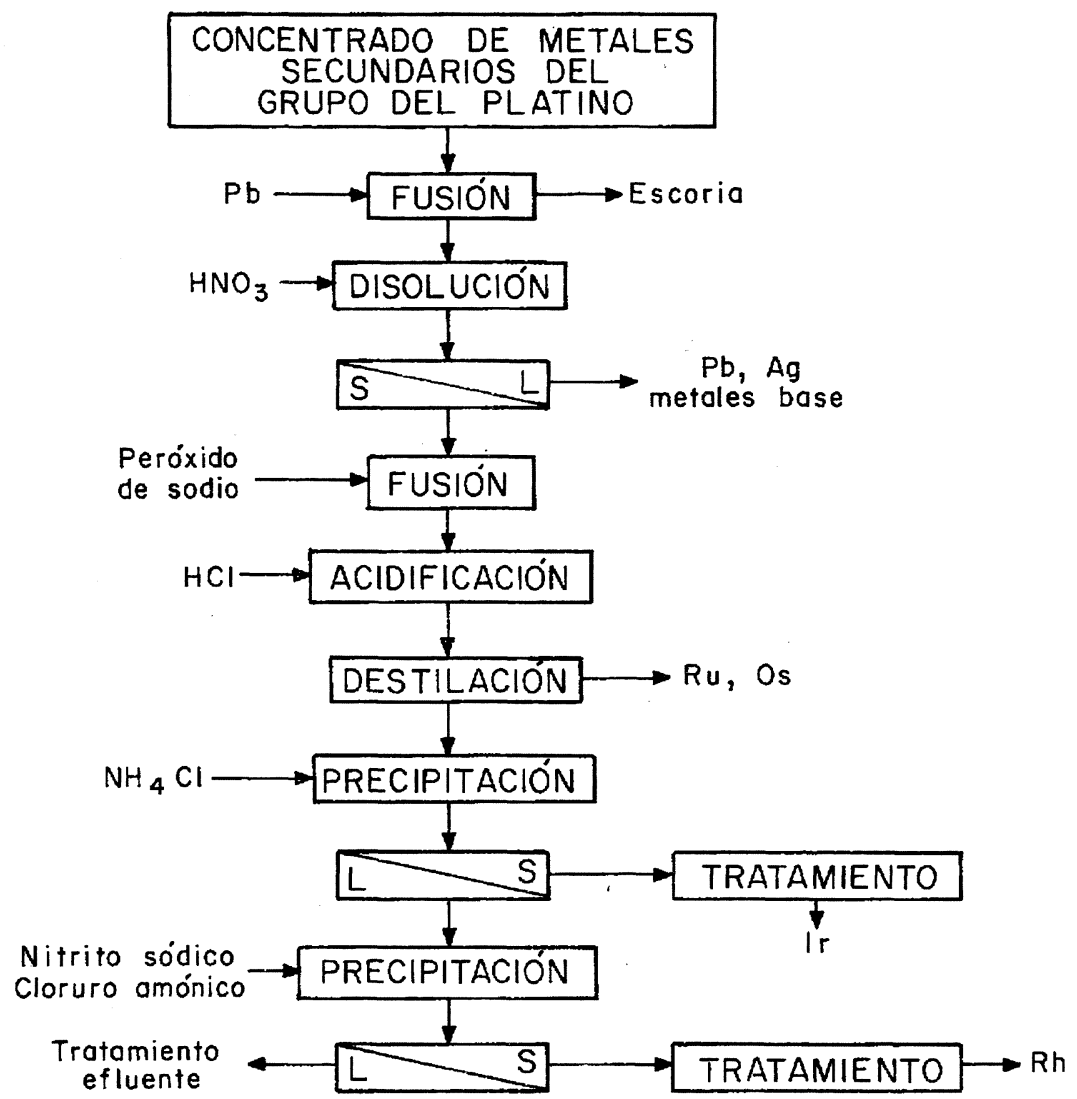

FIG. 2.- Esquema clásico para el refinado de los metales secundarios del grupo del platino.

Fig. 2.-Classical secondary platinum group metals refining scheme.

Este último se trata con peróxido de hidrógeno y se disuelve con ácido clorhídrico, obteniéndose como resultado de estas operaciones unas disoluciones que contienen el Ru(VIII) y el Os(VIII). Los tetróxidos de estos dos metales son muy volátiles, por lo que se recuperan cuantitativamente por destilación recogiéndolos en ácido clorhídrico, donde el rutenio se reduce a $\mathrm{Ru}(\mathrm{VI})$ permaneciendo el osmio como tetróxido, por lo que se puede redestilar y separar.

El iridio y el rodio se separan precipitando al primero de los dos como el complejo hexacloroiridiato de amonio, quedando el rodio en la disolución que precipita en forma del complejo $\left[\mathrm{Rh}\left(\mathrm{NH}_{3}\right)_{5} \mathrm{Cl}\right] \mathrm{Cl}_{2}$.

Estos procedimientos, aunque llevados a la práctica durante años, no son realmente eficientes ya que las distintas etapas de los mismos presentan una serie de desventajas:

- Los procesos son costosos en cuanto al empleo de múltiples etapas de reprecipitación y redisolución hasta que se alcanza el grado de pureza necesario para la comercialización del producto.

- Como consecuencia, el tiempo de procesamiento también se alarga, incluso hasta un año, desde el inicio de la secuencia hasta la obtención de materiales comerciales.
- La necesidad de reciclar en muchas etapas hace que la planta produzca efluentes que contienen metales preciosos, que se recirculan y aumentan el costo del material inmerso en el proceso.

- Las sales cloradas de platino representan un peligro potencial para el operario, debido a su tendencia a producir alergias (6 y 7), lo que hace que las plantas necesiten las medidas de seguridad más extremas, que es otro punto a añadir al coste de la planta.

Por todo ello, se ha hecho precisa la búsqueda de métodos alternativos a los ya descritos, encontrándose en la extracción con disolventes la tecnología que puede resolver, en su mayor parte, la producción de estos metales.

\section{LA EXTRACCIÓN CON DISOLVENTES EN LA RECUPERACIÓN DE LOS METALES DEL GRUPO DEL PLATINO}

Actualmente, la extracción con disolventes es un proceso bien conocido para el beneficio de los metales por sus posibilidades de concentración y separación selectiva en disoluciones acuosas complejas, habiéndose descrito con este fin una serie de 
sistemas de extracción con disolventes o procesos que emplean esta técnica para el tratamiento de disoluciones acuosas que contienen a estos metales preciosos (8).

En el caso de los metales del grupo del platino, se debe tener en cuenta que junto a las propiedades generales que un agente de extracción debe presentar para su aplicación práctica, es muy importante considerar, además, los aspectos de estabilidad de los mismos debido a la naturaleza catalítica de los metales preciosos para dar lugar a reacciones de oxidación, que pueden degradar al reactivo de extracción, y la relativa facilidad con que estos metales pueden reducirse al estado metálico mediante reactivos orgánicos, afectando nuevamente a la estabilidad del agente de extracción.

Por lo tanto, en el refino de los metales preciosos, deben extremarse las exigencias para el uso de un agente de extracción (9):

- Dicho agente tiene que ser muy estable, resistente a procesos de oxidación, y no debe dar lugar a reacciones secundarias que afecten al proceso de extracción.

- Los valores de los correspondientes coeficientes de distribución, $D_{\mathrm{Me}}$, deben ser mayores de 100 , mientras que el proceso de reextracción debe ser también muy eficiente, con $K_{\mathrm{d}}(10)$, mayor de 100.

- La selectividad y la capacidad de carga del agente de extracción deben ser muy altas. En este último caso, de al menos $20 \mathrm{~g} / \mathrm{l}$.

- Los reactivos empleados en la preparación de las disoluciones acuosas de alimentación a las etapas de extracción y reextracción deben ser baratos, y no deben dar lugar a reacciones secundarias.

- Se necesita recuperar fácil y económicamente al metal precioso de la disolución acuosa final. En este caso, es preferible la precipitación en forma de metal o como sal insoluble.

Los nuevos procesos que se han desarrollado para la recuperación de los metales preciosos parecen seguir estos criterios. Algunos de ellos difieren tanto en la filosofía del proceso como en los agentes de extracción empleados, aunque los procesos de extracción son muy selectivos y eficientes (11-18).

\section{LOS NUEVOS PROCESOS PARA EL REFINO DE LOS METALES DEL GRUPO DEL PLATINO}

Aunque muchos productores de metales preciosos han realizado inversiones económicas en la ins- talación de nuevos procesos para el refino del oro, en el caso de los metales relacionados con el platino su recuperación sigue siendo convencional. Aún así, se conocen nuevas refinerías de estos valores metálicos que han adoptado una nueva tecnología basada en la extracción con disolventes, y pese a que, como es lógico, el detalle del proceso es un secreto bien guardado, se van conociendo nuevas características de los mismos.

\subsection{EI proceso INCO}

La figura 3 muestra un esquema de este proceso (19). Como agente de lixiviación de todos los metales preciosos, excepto de la plata, se emplea $\mathrm{HCl} / \mathrm{Cl}_{2}$. De esta forma, se simplifica el esquema global y los metales preciosos se pueden separar mejor. En primer lugar, se recuperan el osmio y el rutenio mediante destilación; esto se debe a la variedad de complejos que puede formar el rutenio que pueden complicar la secuencia de selectividad del proceso, además, de la corriente acuosa principal del esquema se elimina el osmio, muy tóxico, con la consiguiente ventaja que esto conlleva.

A continuación, y si la disolución contiene oro, es necesario recuperar este metal. Esto se debe a que el agente de extracción elegido para el paladio no es selectivo respecto al oro, por lo que este metal se coextraería junto al paladio y, puesto que la reextracción se lleva a cabo con amoniaco, el peligro de formar compuestos inestables de oro-amoniaco es elevado.

La recuperación del paladio se realiza empleando el sulfuro de dioctilo, $25 \% \mathrm{v} / \mathrm{v}$ en ISOPAR M. La cinética de extracción es lenta y, por lo tanto, se opera en discontinuo. La fase orgánica cargada contiene aproximadamente $32 \mathrm{~g} / \mathrm{l}$ de $\mathrm{Pd}$, quedando el refinado con menos de $0,001 \mathrm{~g} / 1$ del metal; la reacción de extracción se puede representar como:

$$
\mathrm{PdCl}_{4 \mathrm{ac}}^{2-}+2 R_{2} \mathrm{~S}_{\text {org }} \Leftrightarrow\left(R_{2} \mathrm{~S}\right)_{2} \mathrm{PdCl}_{2 \mathrm{org}}+2 \mathrm{Cl}_{\mathrm{ac}}^{-}
$$

La fase orgánica cargada se lava con ácido clorhídrico y a continuación se reextrae el metal empleando amoniaco según:

$$
\begin{aligned}
& \left(R_{2} \mathrm{~S}\right)_{2} \mathrm{PdCl}_{2 \text { org }}+4 \mathrm{NH}_{3 \mathrm{ac}} \Leftrightarrow \\
\Leftrightarrow & 2 R_{2} \mathrm{~S}_{\text {org }}+\mathrm{Pd}\left(\mathrm{NH}_{3}\right)_{4 \mathrm{ac}}^{2+}+2 \mathrm{Cl}_{\mathrm{ac}}^{-}
\end{aligned}
$$

La acidificación de la disolución acuosa con $\mathrm{HCl}$ hace que precipite el cloruro de diaminpaladio(II). Esta sal se calcina, o mejor se reduce, con ácido fórmico o formiato de sodio, dando lugar a un metal con una pureza superior al 99,95\%. 


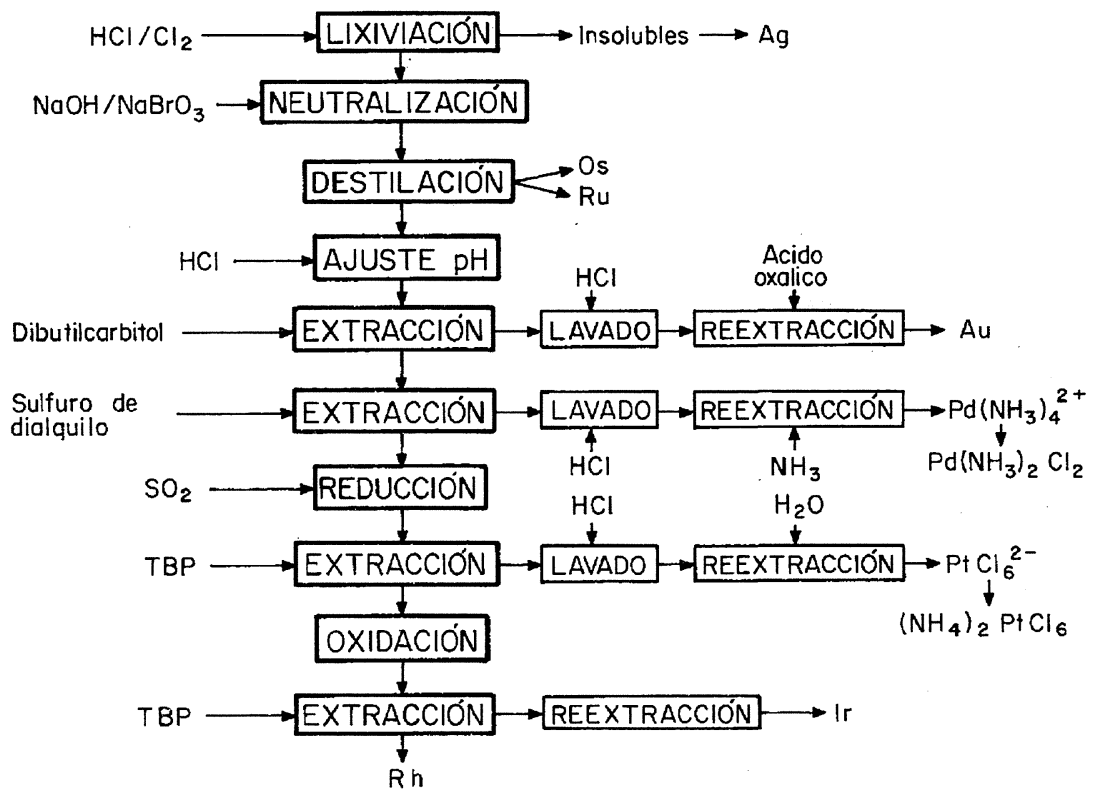

FIG. 3.- El proceso INCO.

Fig. 3.-The INCO process.

El refinado de esta operación de extracción se reduce con $\mathrm{SO}_{2}$ para mantener el iridio como Ir(III), que no se extrae por el TBP, y el platino se recupera mediante este agente de extracción en un medio $\mathrm{HCl}$ de aproximadamente 180 a $215 \mathrm{~g} / \mathrm{l}$. La extracción se lleva a cabo en cuatro etapas en contracorriente, resultando un refinado que contiene de 0,05 a $0,1 \mathrm{~g} / \mathrm{l}$ de Pt. El TBP extrae al platino como $\mathrm{H}_{2} \mathrm{PtCl}_{6}$. La fase orgánica cargada se lava con ácido clorhídrico de la misma concentración que la mencionada con anterioridad, y se reextrae con agua. El platino se recupera de esta disolución precipitándolo como hexacloroplatinato(IV) de amonio, mediante adición de cloruro de amonio. El platino metal se recupera de la sal con una pureza superior al $99,95 \%$.

El refinado se reoxida nuevamente y el iridio se recupera mediante extracción con TBP. El rodio no se extrae, recuperándose ambos metales por precipitación de sus respectivas sales amónicas, seguida de una reducción y/o calcinación. La aplicación de la extracción con disolventes en el caso del iridio, junto a un correcto control de los estados de oxidación, ha supuesto una mejora notable respecto al proceso clásico, puesto que permite la recuperación de este metal sencilla y eficazmente.

\subsection{EI proceso MRR}

En la figura 4 se representa un esquema del proceso MRR. Como en el caso del descrito en el apar- tado anterior, se utiliza una lixiviación global, con $\mathrm{HCl} / \mathrm{Cl}_{2}$, de todos los metales nobles, excepto la plata (20). El oro se recupera mediante dos etapas de extracción con un agente neutro, como la metilisobutil cetona, si bien este agente no es tan selectivo para el oro como el dibutilcarbitol.

El paladio se recupera mediante extracción con disolventes con una $\beta$-hidroxioxima, que necesita operar en discontinuo debido a que presenta una cinética de extracción lenta. Si se emplean mezclas de la oxima con aminas, se puede acelerar la cinética de extracción a costa de disminuir la selectividad del sistema. El mecanismo de actuación de este sistema mixto no se conoce bien, aunque cabe suponer que la extracción transcurre de la siguiente forma:

$$
\begin{aligned}
& \mathrm{PdCl}_{4 \mathrm{ac}}^{2-}+R_{3} \mathrm{NH}^{+} \mathrm{Cl}_{\text {org }}^{-} \Leftrightarrow\left(R_{3} \mathrm{NH}\right)_{2}^{+} \mathrm{PdCl}_{4 \text { org }}^{2-}+ \\
& +2 \mathrm{H} R_{\text {org }} \Leftrightarrow \mathrm{Pd} R_{2 \text { org }}+2 R_{3} \mathrm{NH}^{+} \mathrm{Cl}_{\text {org }}^{-}+2 \mathrm{Cl}_{\text {ac }}^{-}
\end{aligned}
$$

donde $R_{3} \mathrm{~N}$ y $H R$ representan a la amina y a la oxima, respectivamente. Mediante esta reacción, la sal de la amina, $R_{3} \mathrm{NH}^{+} \mathrm{Cl}^{-}$, actúa como catalizador para la transferencia del paladio a la fase orgánica y para la reacción con la oxima. El paladio se reextrae con una disolución de $\mathrm{HCl}$ 5-6 M, y el metal se precipita en forma de la correspondiente sal amónica. La pureza del paladio obtenido, en caso de emplear la oxima, es mayor del 99,9\%, mientras que el empleo del sistema de extracción mixto hace decrecer la pureza del metal hasta algo más del $99,5 \%$. 


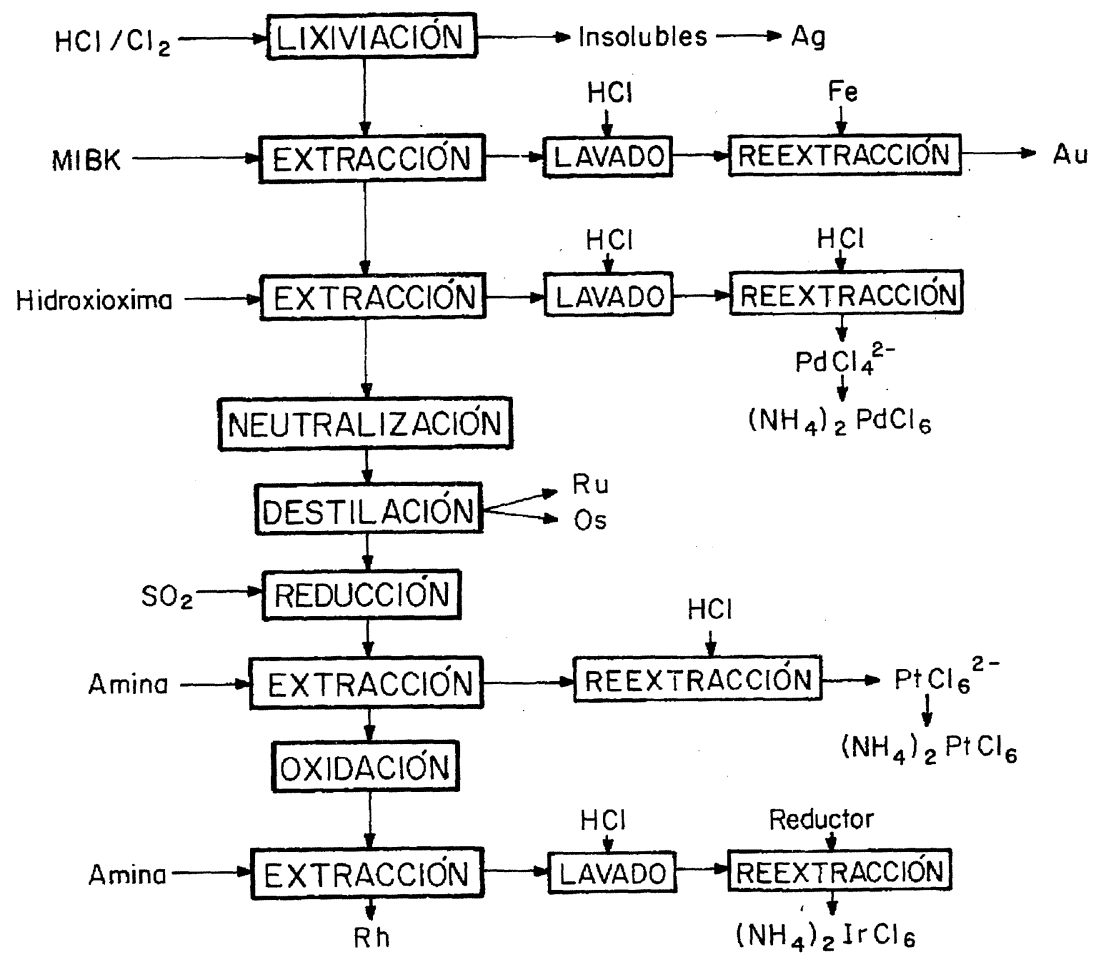

FIG. 4.-El proceso MRR.

Fig. 4.- The MRR process.

El osmio y el rutenio se recuperan mediante destilación de sus tetróxidos y añadiendo un agente oxidante fuerte, como es el bromato de sodio. Los tetróxidos se absorben en una disolución de ácido clorhídrico, el rutenio se reduce a $\mathrm{Ru}(\mathrm{IV})$ y la redestilación conduce a una separación efectiva del osmio.

El platino se recupera mediante extracción con disolventes después de reducir el iridio al estado Ir(III). Como agente de extracción del platino se emplea una amina, preferentemente terciaria, que extrae el metal en forma de $\mathrm{PtCl}_{6}^{2-}$ mediante una reacción de intercambio aniónico. En estos casos existe la posibilidad de que se formen complejos del tipo $\mathrm{Pt}\left(\mathrm{RNH}_{2}\right)_{\mathrm{x}} \mathrm{Cl}_{6-\mathrm{x}}^{+4-(6-\mathrm{x})}$ en la fase orgánica, como consecuencia de los procesos de reextracción, produciendo la acumulación de platino en esta fase y originando toda la problemática que arrastra una situación de esta naturaleza. Hay que hacer notar, sin embargo, que la facilidad de formar este tipo de compuestos disminuye en el orden amina primaria $>$ secundaria $>$ terciaria.

La reextracción se realiza empleando un ácido o un álcali fuerte, recuperándose el platino como el complejo hexacloroplatinato(IV) de amonio.

El refinado se oxida, y el iridio se recupera en forma de Ir(IV), mediante extracción con disolventes, empleando una amina. La fase orgánica se lava con $\mathrm{HCl}$ y el iridio se reduce y reextrae con ácido clorhídrico. Finalmente, el metal se obtiene a partir de la correspondiente sal precipitada. El rodio se puede recuperar mediante una serie de procedimientos, aunque ninguno de ellos es totalmente eficiente.

\subsection{EI proceso MINTEK}

El esquema de este proceso se representa en la figura 5 (2). El agente de lixiviación empleado es nuevamente una mezcla de $\mathrm{HCl} / \mathrm{Cl}_{2}$, recuperándose el oro mediante precipitación con $\mathrm{SO}_{2}$.

El platino y el paladio se extraen simultáneamente mediante un agente de extracción que presenta nuevas características y que se obtiene mediante reacción del ácido cloroacético y la amina secundaria Amberlite LA2. Los dos metales se reextraen simultáneamente con ácido clorhídrico, recuperándose de la disolución acuosa el platino en forma de $\left(\mathrm{NH}_{4}\right)_{2} \mathrm{PtCl}_{6}$. Esta misma disolución acuosa sirve para recuperar el paladio mediante extracción con disolventes con un sulfuro de alquilo, el sulfuro de di-n-hexil. El metal se reextrae con amoníaco y se precipita como la sal clorurada $\operatorname{Pd}\left(\mathrm{NH}_{3}\right)_{2} \mathrm{Cl}_{2}$. En el caso del paladio, se obtiene una pureza del 99,96 al $99,99 \%$, mientras que para el platino es del $99,9 \%$.

El osmio se recupera por destilación. 


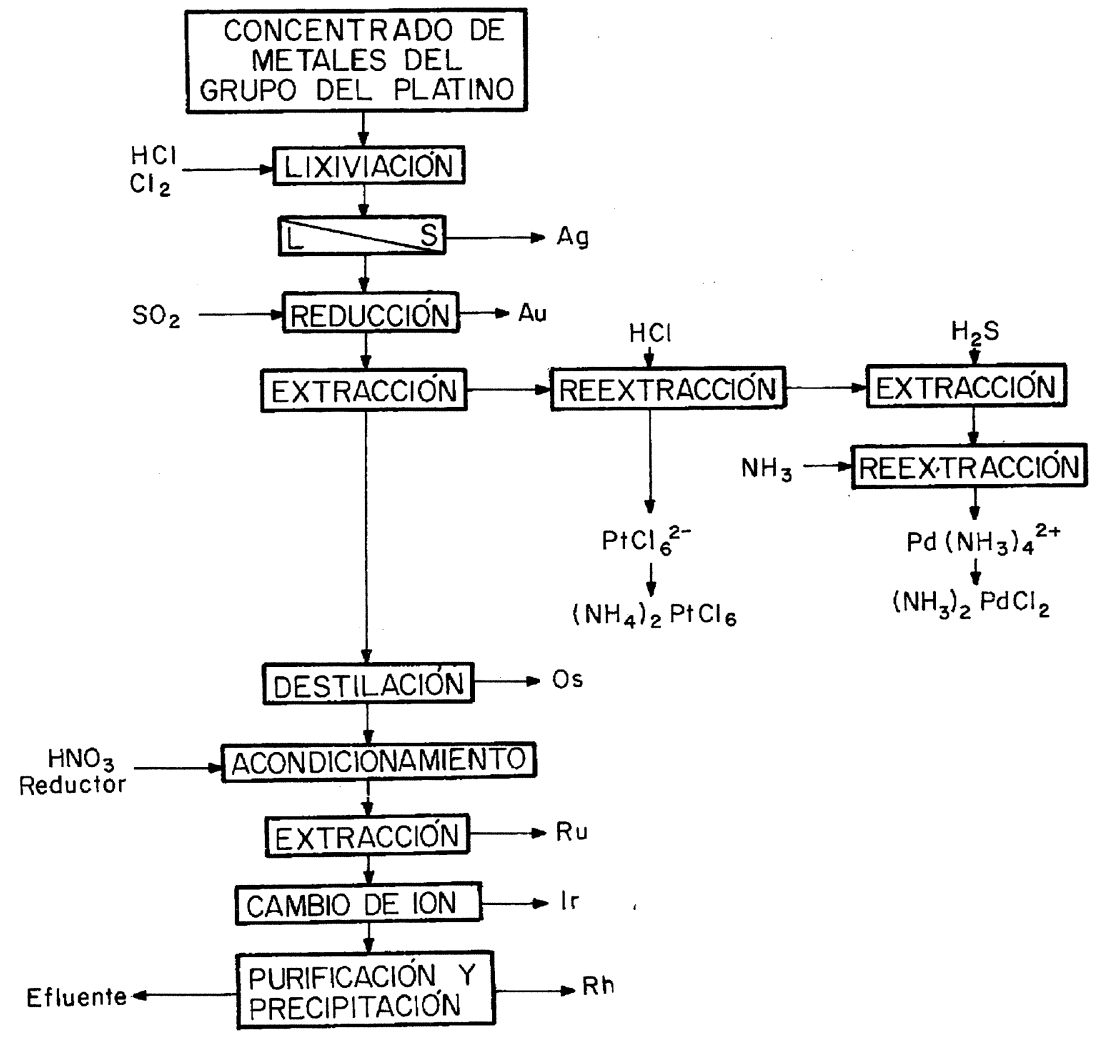

FIG. 5.-El proceso MINTEK.

Fig. 5.- The MINTEK process.

La disolución acuosa resultante se estabiliza con la adicción de los ácidos nítrico y fórmico, para el rutenio, o bisulfito de sodio e hidroxilamina, para reducir al iridio a $\operatorname{Ir}(\mathrm{III})$; el rutenio se recupera mediante extracción con disolventes empleando una amina terciaria. La reextracción se lleva a cabo con una disolución de sosa y, después de acidificar con $\mathrm{HCl}$, el rutenio se precipita como el complejo hexaclororutenato(IV) de amonio mediante la adicción de cloruro de amonio. El metal se obtiene, finalmente, a partir de esta sal.

El iridio se recupera en una etapa de intercambio aniónico con una resina, Amberlite IRA 400, llevándose a cabo la elución con agua saturada con $\mathrm{SO}_{2}$. Se necesita una etapa de purificación posterior que se realiza con TBP. Por último, el rodio se recupera mediante precipitación de su sal amoniacal.

\subsection{EI proceso LONRHO}

Una variante al proceso MINTEK es el que se describe a continuación, representándose en la figura 6 el esquema del mismo. Las etapas de separación-recuperación de los metales hasta la etapa de estabilización de la corriente acuosa principal en este nuevo proceso, es similar al esquema mostrado en la figura 5.
En el proceso LONRHO, el rodio se recupera a continuación por extracción con disolventes con una amina y por reextracción con $\mathrm{NaOH}$, aunque también se ha propuesto la tiourea como agente de reextracción para este metal (21). El rodio se obtiene en forma de $\mathrm{Rh}_{2} \mathrm{O}_{3}$.

El rutenio, estabilizado como $\left[\mathrm{RuNOCl}_{5}\right]^{2-}$, por ejemplo, se extrae con una amina terciaria, se reextrae con hidróxido sódico y se recupera como el óxido correspondiente.

Del refinado obtenido de esta operación, una vez oxidado, se extrae el iridio con TBP, se reextrae con agua precipitando la sal $\left(\mathrm{NH}_{4}\right)_{2} \mathrm{IrCl}_{6}$, y se reduce y/o calcina esta última.

\section{OTRAS PROPUESTAS PARA LA RECUPERACIÓN DE LOS METALES DEL GRUPO DEL PLATINO}

Junto a los procesos descritos en el apartado anterior, se han propuesto otros procedimientos para la recuperación de estos metales. Estas propuestas están basadas, sobre todo, en el empleo de la extracción con disolventes.

En el caso del paladio, se propone la extracción de este metal con agentes que contienen átomos de 


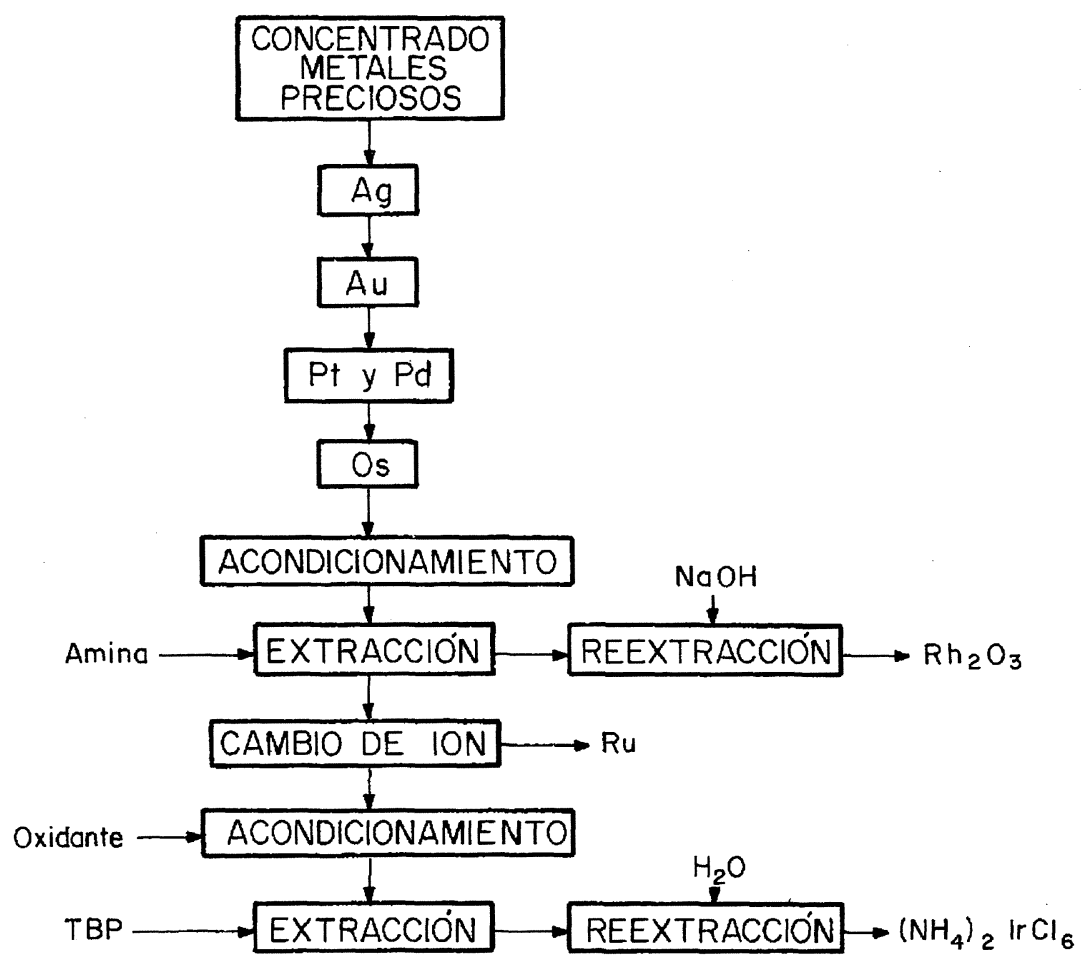

FIG. 6.- El proceso LONRHO.

Fig. 6.- The LONRHO process.

azufre y oximas (22-26). También extraen este metal los derivados orgánicos del ácido fosfórico (27-29), sin embargo, la etapa de reextracción se realiza con dificultad y es necesario emplear disoluciones recientemente preparadas de tiosulfato de sodio. Aunque estos últimos agentes presentan buenas características, no son comparables, en cuanto a la facilidad para llevar a cabo la reextracción, con las oximas y los derivados orgánicos con átomos de azufre.

Otra propuesta se basa en el empleo de la dimetilglioxima como agente precipitante selectivo del paladio de disoluciones en medio nitrato. Estas disoluciones provienen del tratamiento de lodos anódicos argentíferos con ácido nítrico. Como se ha mencionado, esta precipitación es selectiva respecto a la presencia de paladio y rodio en la disolución acuosa (30).

Algunos derivados comerciales de la quinolina, como los conocidos con el nombre de LIX 26 (Henkel) y TN 1911 (Schering), se pueden utilizar en la recuperación de platino y paladio (31-36). Este último reactivo presenta buenas características cinéticas respecto a la extracción y reextracción del paladio. La reextracción se lleva a cabo con $\mathrm{HCl} 6$ $8 \mathrm{M}$, que al no necesitar amoniaco en esta etapa, puede beneficiar la descarga de efluentes en determinadas situaciones. Asimismo, este agente de extracción permite la consecución de productos finales de alta pureza (casi no se extraen otras impurezas) y, en resumen, propicia la extracción conjunta de estos dos metales preciosos en una operación continua.

También se ha propuesto un esquema de tratamiento que sólo emplea aminas para la recuperación de estos metales nobles. El mayor inconveniente de éste es que necesita diversos agentes de reextracción con disoluciones acuosas, tales como tiourea o sulfocianuro de sodio, para la recuperación de estos metales preciosos. La preparación de estas disoluciones no es fácil $\mathrm{y}$, por otro lado, las aminas son, a veces, difíciles de reextraer y tienden a presentar cinéticas de reextracción lentas.

Los únicos esquemas de tratamiento propuestos, que no emplean la extracción con disolventes, utilizan, en un caso, la fluorinización de los metales nobles (37 y 38) formándose especies volátiles que se pueden condensar en una etapa posterior. Este esquema presenta la ventaja de que se consigue una separación selectiva del paladio, debido a que este metal no forma con facilidad un fluoruro volátil. Para que el proceso alcance los mejores resultados, es necesario formar los penta o hexafluoruros de estos metales.

El otro esquema de tratamiento emplea un derivado de la tiourea para la precipitación selectiva y global de los metales preciosos. La ventaja de este 
procedimiento es que con él se obtiene un concentrado puro de estos metales que facilita su tratamiento posterior (39).

Las posibles ventajas de estos dos últimos esquemas quedan disminuidas por el hecho de que los fluoruros son difíciles de emplear en la práctica y porque la tiourea y sus derivados pudieran ser sustancias cancerígenas.

La recuperación del rodio e iridio también ha sido un tema que ha despertado interés, con aportación de nuevas propuestas encaminadas a tal fin.

El iridio se puede recuperar directamente de disoluciones de ácido clorhídrico mediante una reducción con hidrógeno (40). La separación de ambos elementos también se puede llevar a cabo con amidas $\mathrm{N}$-sustituidas o con TBP (41 y 42). En este último caso, es necesario evitar el uso del agua regia, debido a la tendencia del iridio a formar determinados complejos con el nitrato, lo que hace que la extracción no sea completa. Sin embargo, el uso de peróxido de hidrógeno como oxidante, hace que la extracción del metal sea del $100 \%$.

En el caso del rodio se ha descrito un nuevo proceso basado en el empleo del reactivo TN 1911, ya mencionado con anterioridad (43). En dicho proceso es necesario estabilizar el metal en la disolución acuosa antes del proceso de extracción. La reextracción también es una operación que se lleva a cabo con gran eficiencia. Otros nuevos sistemas, empleados para la recuperación de este metal de disoluciones purificadas, utilizan la dinonilglicina, la cementación con cobre y la extracción con derivados organofosforados (44-46).

Un último avance en la recuperación de estos metales preciosos ha sido la aplicación del principio de la tecnología de reconocimiento molecular, o MRT (47-50). Como agentes orgánicos se emplean éteres corona, que si por una parte son unos reactivos conocidos desde hace tiempo en química orgánica, por la otra sólo muy recientemente la ingeniería orgánica ha logrado que sean agentes efectivos para el reconocimiento específico de moléculas o iones en disolución.

El empleo de estos nuevos sistemas puede cambiar el concepto de recuperación de los metales preciosos, aunque todavía existen determinados problemas que no están resueltos. Por ejemplo, se necesita encontrar agentes de elución que sean comerciales que se puedan también aceptar desde el punto de vista medioambiental. Se han propuesto como tales la etilendiamina, las disoluciones acidificadas de tiourea y el $\mathrm{HCl}$ concentrado. Otro aspecto a tener en cuenta es la necesidad de encontrar soportes inertes y estables para fijar a estos nuevos reactivos. Desde el punto de vista de estos soportes, es necesario, por el momento, evitar el uso de agentes oxidantes enérgicos, tales como el cloro, que degradan a dichos soportes; además, la utilización de un valor de $\mathrm{pH}$ por encima de 10 , tiende también a destruir el reactivo orgánico.

En el caso del paladio, el eluyente ha de tener concentraciones del orden de $5 \mathrm{~g} / \mathrm{l}$, debiendo hallarse el paladio en el estado de $\operatorname{Pd}(\mathrm{IV})$. La etapa de reextracción o elución se debe hacer lo más rápidamente posible, puesto que dicho elemento tiende a reducirse en el sistema.

Para el caso del rodio, los condicionamientos son similares, aunque el empleo de sistemas con estas características hace que este metal se pueda recuperar al inicio de los esquemas de tratamiento, hecho que no deja de ser una ventaja teniendo en cuenta el elevado precio del metal.

\section{CONCLUSIONES}

Los procesos de recuperación de los metales del grupo del platino, aunque empleados en la práctica desde hace años, presentan una serie de desventajas que se pueden resumir en que son largos, tediosos y producen numerosos efluentes que a la vez contienen a estos metales preciosos, con lo que un volumen de los mismos, que puede ser importante, se encuentra recirculando por el circuito. Además, teniendo en cuenta las nuevas restricciones de seguridad, parece que existe una determinada prevención hacia la obtención de compuestos tales como las sales cloradas de platino, que son tóxicas.

Todo ello hace que se haya desarrollado una nueva tecnología de recuperación de estos metales, basada, principalmente, en el empleo de la extracción con disolventes. El éxito de tales procesos ha hecho que, al menos tres o cuatro de los mismos, tengan una firme implementación comercial. Las ventajas encontradas en estos nuevos procedimientos se resumen también en que: los metales preciosos se disuelven en una etapa; la recuperación alcanza, en la mayoría de los casos, el $100 \%$ del metal en una sola etapa y, por lo tanto, se elimina la acumulación de éstos en los circuitos; presentan gran flexibilidad operacional, siendo también más segura, desde este punto de vista, la manipulación de compuestos tóxicos de platino y/o osmio, $y$, finalmente, disminuyen los costes operacionales y de capital.

Además, estos avances han hecho que, paralelamente, se estén desarrollando nuevos sistemas de recuperación que, como en el caso de los procedimientos MRT, permiten la obtención de estos metales preciosos de disoluciones diluidas. 


\section{REFERENCIAS}

(1) Alguacil, F.J. Rev. Metal. En prensa, 1995.

(2) EdwARDS, R.I. y TE RIELE, W.A.M. Handbook of Solvent Extraction. Eds. T. Lo, M.H.I. BAIRD y C. HANSON. Wiley-Interscience. Nueva York, 1983.

(3) Edwards, R.I. y Te Riele, W.A.M. Platinum Supplement. Vol. A1. Gmelin Handbook of Inorganic Chemistry. Springer Verlag. Heidelberg, 1986.

(4) FuwA, A. Metallurgical Review of MMIJ, 4 (1), 1987: 98115.

(5) Foo, G.J. J. Metals, 34 (10), 1982: 59-62.

(6) Cleare, M.J., Hughes, E.G., Jacoby, B. y Pepys, J. $J$. Clinical Allergy, 6, 1976: 183.

(7) BRADFORD, C.W. Precious Metals 1991. Ed. L. Manziek, IPMI. Naples, 1991.

(8) AMER, S. Rev. Metal, 19 (3), 1986: 161-183.

(9) Edwards, R.I. J. Metals, 28 (8), 1976: 4-9.

(10) Alguacil, F.J., Caravaca, C., Cobo, A., y Martinez, S. Hydrometallurgy, 35, 1994: 41-52.

(11) Edwards, R.I. Proceedings ISEC 77. CIM Special Vol. 21. Montreal, 1979.

(12) Cleare, M.J., Charlesworth, P. y Bryson, D.J. J. Chem. Tech. Biotechnol., 29, 1979: 210-224.

(13) Reavill, L.R.P. y Charlesworth, P. Proceedings ISEC 80. Lieja, 1980.

(14) Charlesworth, P. Platinum Metals Review, 25 (3), 1981: 106-112.

(15) Cleare, M.J., Grant, R.A. y Charlesworth, P. Extraction Metallurgy'81. IMM. Londres, 1981.

(16) Edwards, J.D., Colton, D.F. y Lea, R.K. Pat. Europea 49567, 1982.

(17) Lea, R.K., Edwards, J.D. y Colton, D.F. Pat. EE.UU. 4390366, 1983.

(18) Grant, R.A. Precious Metals Recovery and Refining. Ed. L. Manziek. IPMI. Nueva York, 1990.

(19) Barnes, J.E. y Edwards, J.D. Chem. Ind., Mar., 1982: 151-155.

(20) Grant, R.A. y Murrer, B.A. Pat. Europea 210004, 1987.

(21) DHARA, S.C. Precious Metals Mining, Extraction and Processing, Eds. V. Kudryk, D.A. Corrigan y W.W. Laing. Met. Soc. of AIME. Warrendale, 1984.

(22) Inoue, K. y MaruUchi, T. Hydrometallurgy, 16 (1), 1986: 93-104.

(23) Inoue, K. y BABA, Y. Proceedings ISEC 86. Ed. Dechema. Frankfurt, 1986

(24) Inoue, K., BABA, Y. y OKA, T. Hydrometallurgy, 18 (2), 1987: 265-272.

(25) Koshimura, K. Chem. Abstr., 109: 41250u, 1988.

(26) Bauer, D., Cote, G. y DaAmach, S. Chem. Abstr., 112: 183320c, 1989.

(27) Wenjun, C., Yunlong, W. y Jiezhen, L. Proceedings First International Conference on Hydrometallurgy. Eds.
Z. Yulian y X. Jlazhong. International Academic Pub. Pekín, 1988.

(28) Walker, R.D. y Bautista, R.G. Proceedings ISEC 86. Ed. Dechema. Frankfurt, 1986.

(29) ICHISHI, T. Chem. Abstr., 110: 139100, 1988.

(30) Hermann, S., Schubert, F. y Zaborowski, B. Chem. Abstr., 110: 139131b, 1988.

(31) MA, E. y Freiser, H. Inorg. Chem., 23 (21), 1984: 33-44.

(32) Demopoulos, G.P. J. Metals, Jul., 1986: 13-17.

(33) Pouskouleli, G., KelebeK, S. y Demopoulos, G. P. Separation Processes in Hydrometallurgy. Ed. G. Davies. Ellis-Horwood. Chichester, 1987.

(34) Demopoulos, G.P., KuyucaK, N., Gefvert, D.L. yं RitCHER, M.H. Precious Metals 1989. Ed. B. Harris. IPMI. Allentown, 1989.

(35) Harris, G.B., Monette, S., Barry, J.-P. y Stanley, R.W. Pat. EE.UU. 5045290, 1991.

(36) Harris, G.B., Monette, S. y Vleeschhouwer, D. Hydrometallurgy: Fundamentals, Technology and Innovation. Ed. AIME-SME. Littleton, 1993.

(37) Boyd, D.A., Holloway, J.H. y Stanger, G.Z. Pat. Europea 330335, 1989.

(38) BoyD, D.A. Precious Metals 1991. Ed. L. Manziek. IPMI. Naples, 1991.

(39) Konig, K.H., Schuster, M., Hollman, D. y Schlodder, R. Pat. Canadá 1230976, 1988.

(40) Xiansheng, N., Jing, C. y Qinglin, T. Precious Metals 1989. Ed. B. Harris. IPMI. Montreal, 1989.

(41) Grant, R.A., Burnham, R.F. y Collard, S. Solvent Extraction 1990. Part A. Ed. T. Sekine. Elsevier. Amsterdam, 1992.

(42) JING, C. Precious Metals 1991. Ed. L. Manziek. IPMI. Naples, 1991.

(43) Demopoulos, G.P. y Benguerel, E. Rhodium/Sampling and Analysis. Eds. R.C. Kaltenbach y L. Manziek. IPMI. Orlando, 1992.

(44) DaI, N., Deng, D., WANG, J. y Hu, X. Chem. Abstr., 104: 210490b, 1987.

(45) Jing, C., Ning, C. y Zhengfen, Y. Proceedings First International Conference on Hydrometallurgy. Ed. Z. Yulian y X. Jiazhong. International Academic Pub. Pekín, 1988.

(46) Xiangyun, W., Ming, J., Xingi, L. y Yuanfang, L. Íbid.

(47) Ruckman, J.H. Precious Metals 1989. Ed. B. Harris. IPMI. Montreal, 1989.

(48) Wright, C. y Breuning, R.L. Precious Metals Recovery and Refining. Ed. L. Manziek. IPMI. Scottsdale, 1989.

(49) Breuning, R.L., Izatt, S.R. y Griffin, L.D. Precious Metals 1990. Ed. D.A. Corrigan. IPMI. San Diego, 1990.

(50) IChishi, S., Griffin, L.D. y Breuning, R.L. Rhodium/Sampling and Analysis. Eds. R.C. Kaltenbach y L. Manziek. IPMI. Orlando, 1992. 